\title{
How crucial is it to account for the antecedent moisture conditions in flood forecasting? Comparison of event-based and continuous approaches on 178 catchments
}

\author{
L. Berthet ${ }^{1,2}$, V. Andréassian ${ }^{1}$, C. Perrin ${ }^{1}$, and P. Javelle ${ }^{3}$ \\ ${ }^{1}$ Cemagref, Hydrosystems and Bioprocesses Research Unit Antony, France \\ ${ }^{2}$ AgroParisTech ENGREF, 19 avenue du Maine, 75732 Paris, France \\ ${ }^{3}$ Cemagref, Hydrology and Hydraulic Works Research Unit, Aix-en-Provence, France
}

Received: 17 February 2009 - Published in Hydrol. Earth Syst. Sci. Discuss.: 5 March 2009

Revised: 27 May 2009 - Accepted: 2 June 2009 - Published: 18 June 2009

\begin{abstract}
This paper compares event-based and continuous hydrological modelling approaches for real-time forecasting of river flows. Both approaches are compared using a lumped hydrologic model (whose structure includes a soil moisture accounting (SMA) store and a routing store) on a data set of 178 French catchments. The main focus of this study was to investigate the actual impact of soil moisture initial conditions on the performance of flood forecasting models and the possible compensations with updating techniques. The rainfall-runoff model assimilation technique we used does not impact the SMA component of the model but only its routing part. Tests were made by running the SMA store continuously or on event basis, everything else being equal. The results show that the continuous approach remains the reference to ensure good forecasting performances. We show, however, that the possibility to assimilate the last observed flow considerably reduces the differences in performance. Last, we present a robust alternative to initialize the SMA store where continuous approaches are impossible because of data availability problems.
\end{abstract}

\section{Introduction}

1.1 Continuous vs. event-based approaches to modelling

From the catchment point of view, the hydrological cycle is a sequence of wetting and drying periods. On a given date, the

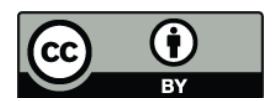

Correspondence to: L. Berthet (lionel.berthet@cemagref.fr) moisture state of a catchment is the consequence of the past sequence of meteorological conditions. The initial moisture conditions at the beginning of a rainfall event have a major influence on a catchment's hydrological response. Therefore the set-up (as defined by Refsgaard and Henriksen, 2004) of a hydrological model requires choosing the initial conditions. Depending on how this is done, hydrological models will be categorized as continuous or event-based.

The initialization through a continuous approach consists in running the model during a warm-up period in order to let the model states reach values that no longer depend on arbitrarily chosen initial values. The duration of this warmup depends on the catchment (its memory of past conditions) and on the model and may last a few months (Kitanidis and Bras, 1980b). A climatic cycle (i.e., one year) is often used, although it has been shown that some catchments (especially those where large aquifers feed streamflow) need up to several years (Le Moine, 2008). In an operational forecasting perspective, the major drawback of the continuous approach lies in its data requirements: long continuous precipitation time series up to the day of interest are difficult to provide (data gaps occur frequently because of real-time data repatriation difficulties). Another consequence is the necessity of having to gather a long enough data series before issuing the first forecast at new locations.

In contrast, event-based models require a separate method to derive the initial values of model states. Numerous methods exist. If the model states reliably represented measurable physical quantities, recent measurements or values based on climatology would be solutions. For example, Brocca et al. (2009) showed that assimilating soil moisture measurements into the event-based SCS-CN model can be useful for flow

Published by Copernicus Publications on behalf of the European Geosciences Union. 
simulation on a small catchment. However, these results should be generalized, as mentioned by the authors.

Continuous approaches have been recommended to modellers for many years (e.g. Kitanidis and Bras, 1980a; Linsley, 1982) as a rigorous solution to the estimation of initial conditions. However, we must acknowledge that event-based approaches are still often preferred in real-time operational applications (Lamb and Kay, 2004). Event-based models may be simpler because they often do not need to include all the processes necessary in a continuous model. This means more limited data requirements which may ease model implementation and use. Another reason lies in the difficulty maintaining and validating automatic measurement networks over a long period in many countries. This is a frequent situation when looking for high time resolution series. To bypass this obstacle, Nalbantis (1995) suggests relying on coarser data series (e.g. daily) to estimate fine (hourly) initial conditions. The problem may also be cultural. Some endusers, who traditionally use hydraulic propagation methods, are culturally in favor of an event-based approach. Despite all the good reasons advanced by hydrologists for using continuous approaches, practitioners often continue using eventbased models and see them as the only solution. Another reason in favor of the event-based modelling is raised when the model is not used only for flood forecasting (e.g., for other uses like torrential flood modelling).

\subsection{Sensitivity of hydrological models to the initializa- tion procedure}

The report of the National Research Council (NRC) (2002) identified as crucial the question of initial conditions. There is a wide consensus among hydrologists that hydrological models' outputs are very sensitive to initial conditions, especially soil moisture or catchment wetness (e.g. Refsgaard et al., 1999; Moore et al., 2006; Vivoni et al., 2007). Eventbased models can lead to very different outputs when run with different initial conditions (Da Ros and Borga, 1997). As hydrological processes are essentially non linear, even a slight uncertainty on initial conditions can lead to dramatic uncertainty on streamflow (Zehe and Blöschl, 2004).

Many authors have studied the effects of initialization on the response of models that seek to reproduce physical processes. Already at the inception of the Soil Conservation Service (SCS) Curve Number $(\mathrm{CN})$ formula, modulating the $\mathrm{CN}$ value according to the antecedent moisture conditions has been found to be necessary (Ogrosky and Mockus, 1964). More recently, Noto et al. (2008) showed that the degree of sensitivity to the initialization procedure depends on other factors, such as the intensity of precipitation or the catchment's physical properties. Vieux et al. (2004) demonstrated that the sensitivity of the model is lower when the catchment is already very wet.

\subsection{The real-time forecasting specificities}

The sensitivity of hydrological models to initial conditions is of prime importance for operational forecasting. For example, Norbiato et al. (2008) showed that initial conditions (antecedent soil moisture) are essential for efficient flash flood alerts.

Real-time forecasting systems most often use a data assimilation method to improve short-range prediction accuracy (Shamseldin, 2006). Among the different assimilation techniques, state updating is quite popular (Refsgaard, 1997; Moore, 2007). This method estimates state variables depending on the very last observed discharges. Consequently, the question of initial conditions appears to be less important if some (or even all) states are re-estimated by this updating technique (Nalbantis, 2000; Aubert et al., 2003; Moore et al., 2005).

State updating, when performed by assimilation of a small number of measured inputs (e.g. only discharge or discharge and soil moisture) compared to the number of internal states, leads to uncertainties which combine with the uncertainty on the initial values. Indeed, trying to update several model states simultaneously may endanger model robustness and it leaves the modeller in the uncomfortable situation where there are more unknowns than equations to solve.

The uncertainties due to the initial conditions may also be taken into account by using an ensemble forecast whose members differ in their initial conditions (e.g. Dietrich et al., 2008). However, this issue is not within the scope of this paper and we will focus on deterministic forecasting methods for the sake of simplicity.

\subsection{Scope of the paper}

This paper has both a theoretical and an applied objectives. The theoretical one is to contribute to a more general answer to the relative merits of continuous and event-based approaches for flood forecasting, through the comparison of different initialization approaches for the very same flood forecasting model. Indeed, although this issue has long been in the forefront, the literature does not provide any clear answer to this question. In addition, we investigate the possible interplay between the updating techniques and the initialization impact: the applied objective is to determine whether we can define simple initialization schemes which allow issuing forecasts without running the model over a long preforecast period. Initialization strategies are tested on a set of 178 French catchments.

Several authors compared different models with different initialization strategies. For example, Amengual et al. (2008) compared the performances of two different models - one being continuous, the other one event-based - to hindcast a flash flood event and found little difference between them. Instead, we choose to use the very same model in order to focus exclusively on the initialization strategies. 


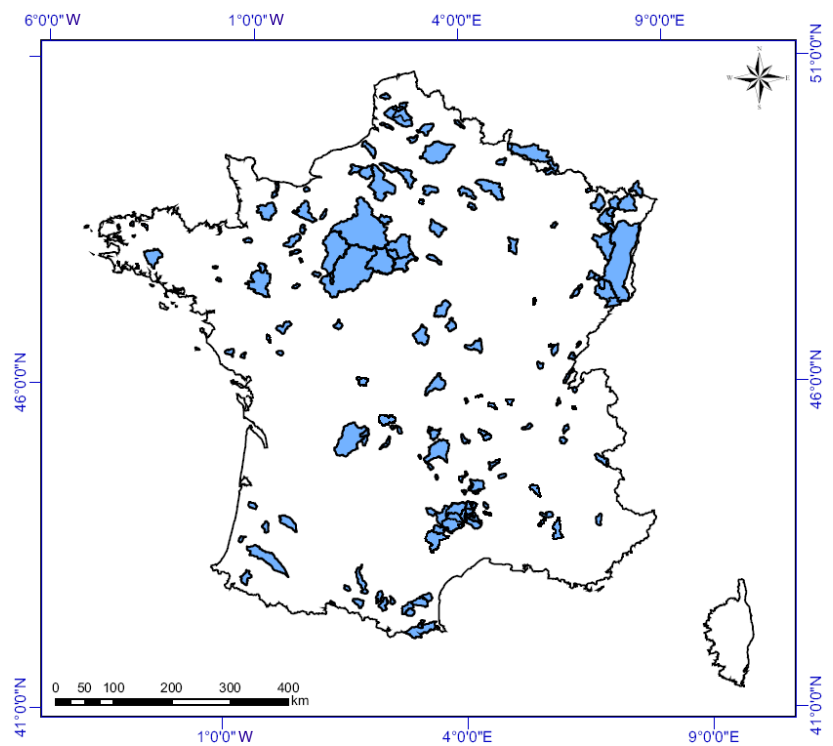

Fig. 1. Locations of the 178 French catchments used in this study.

The remainder of the article is organized as follows: first the data and the model are described as well as the assessment criteria we employed. Then Sect. 3 details the methodology. The results are shown and discussed in Sect. 4. Finally a number of conclusions are drawn.

\section{Catchments set, model and assessment criteria}

\subsection{Test set of 178 French catchments}

The comparison is based on 178 French unregulated catchments (Fig. 1), chosen to represent the hydroclimatic variability encountered in the country (note, however, that no selected catchment is significantly snow-affected and requires a snowmelt module to ensure a proper modelling). Catchment areas range from 10 to $5940 \mathrm{~km}^{2}$ (average of $354 \mathrm{~km}^{2}$; median of $125 \mathrm{~km}^{2}$ ). By working on various catchments, we aim to ensure more general conclusions to our study (Andréassian et al., 2006). Our data set covers a varied range of hydrological behaviours: some Mediterranean catchments experience flash floods, whereas others typically have slow floods. We used continuous hourly precipitation, discharge and potential evapotranspiration (PE) data series from 1995 to 2005. PE values were computed using the formula proposed by Oudin et al. (2005), based on temperature and extra-atmosphere global radiation (Morton, 1983). The quality of data used in this study was controlled and is supposed higher than the quality of data used in real-time operations.
SMA store to be initialized by continuous run or a specific event method

Store initialized by assimilation of the last observed streamflow (updating)

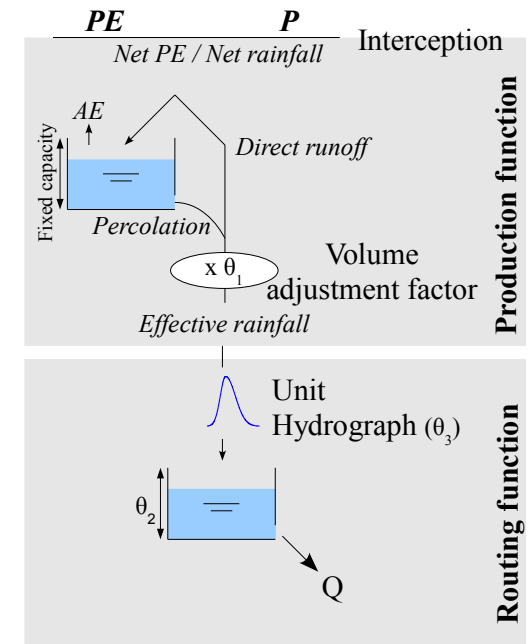

Fig. 2. Structure of the GRP model. The SMA store has to be initialized while the routing store level is updated when used in forecasting mode. $P E$ and $A E$ are potential and actual evapotranspiration. $\theta_{i}$ are model parameters: a volume adjustment factor, the capacity of the routing store and the base time of the unit hydrograph.

\subsection{Forecasting model}

Our objective was to compare different initialization modes using the very same model structure. We deliberately used a simple model (GRP) in order to unambiguously analyze the effects of different initializations. It is an efficient operational model, one of those used to forecast river flows in real time on the Seine basin upstream from Paris (Cemagref, 2005). Detailing the structure of the forecasting model is not within the scope of this paper; therefore, only a brief description follows.

GRP is a hybrid metric-conceptual lumped parsimonious model, designed specifically for flood forecasting (see Tangara, 2005, for mathematical details). Its structure was derived from the structure of the GR4J model (Perrin et al., 2003). GRP can classically be described as a production function followed by a routing function (Fig. 2). The production function consists in a non-linear "soil moisture accounting" (SMA) reservoir and a volume adjustment coefficient which determine the runoff ratio. The SMA store requires either a specific initialization or a continuous running mode. The routing function is composed of a unit hydrograph (UH) and a non-linear routing store.

The forecasting model GRP uses a combination of two assimilation (updating) functions for flood forecasting. The first exploits the last observed discharge information to update the state of the model routing store, while the second draws information from the last model error to update the model's output through a multiplicative ARIMA model (Box and Jenkins, 1976). We do not use the Kalman filter (or one of its heirs) because we found it could lead to performance 
losses during flood events when it assimilates streamflow alone (Aubert et al., 2003). The important thing to note here is that the level of the SMA store is not updated in the model and its initialization will be the main focus of the tests presented hereafter.

The model includes two main state variables: the levels of the production store and of the routing store. We do not consider the internal states of the unit hydrograph since they are very transient states: their values no longer depend on their initial values after a finite number of time steps because the model UH has a finite number of ordinates.

\subsection{Assessment criteria}

For a given lead time $L$, the overall evaluation of the forecasts is based on the persistence index PI (Kitanidis and Bras, 1980a):

$$
P I(L)=1-\frac{\sum_{t}\left(\widehat{Q}_{t+L \mid t}-Q_{t+L}\right)^{2}}{\sum_{t}\left(Q_{t}-Q_{t+L}\right)^{2}}
$$

where $Q_{t}$ and $Q_{t+L}$ are the observed discharge at time step $t$ and $t+L$, respectively, while $\widehat{Q}_{t+L \mid t}$ is the forecast issued at time step $t$ for time step $t+L$. A PI value of 1 indicates a perfect fit between forecasted and observed discharges. A positive value means that the root mean square error (RMSE) of the assessed model is lower than the RMSE of the persistence model which gives the last observed discharge as a prediction for the future time steps. A negative value implies that the model is less efficient than the persistence model. The criterion value mostly reflects performances during floods since it is a quadratic criterion. The PI is a well-suited quadratic criterion to assess forecasting models, since it compares the tested model to a naive one that uses the same information of observed discharge.

In addition, we used a time criterion to assess the time difference between the observed and forecasted flood events. We are interested in the time-to-peak delay (or the time to a fraction - say $90 \%$ - of the peak). Therefore we considered the mean time-to-peak delay for the identified flood events.

Last, the visual comparison of observed and forecasted hydrographs for significant events will complete our analysis.

\section{Methodology}

\subsection{Modus operandi}

Since only the effects of the initialization procedure are to be assessed, we used the same model and the same sets of parameters for the various initialization methods we tested. For each catchment, the model was calibrated by an automatic algorithm in a continuous mode (over a continuous series of 5 years). The PI was used as the objective function for parameter calibration. Even if the effects of the calibration approach (continuous or event-based) are not insignificant for the overall performance of the model (see e.g. Tan et al., 2008), we do not discuss this aspect here for the sake of brevity.

Flood forecasting requires future precipitation scenarios. In real-time conditions, some quantitative precipitation forecasts (QPF) may be available. In our study, we adopted a perfect foreknowledge scenario: this scenario corresponds to observed precipitations for the future time steps. While this is clearly not a realistic scenario in real-time conditions (it is overly optimistic), we selected this approach because we wish to focus the analysis on the effect of the initial conditions without adding other sources of uncertainty.

We used a classical split-sample test scheme (Klemeš, 1986) to assess the model's versions. The complete 10-year record available on every catchment was split into two 5-year sub-periods that alternatively served for calibration and validation. Only the results obtained on validation periods are shown.

Different catchments may have very different responses. To check whether different initialization solutions could fit different types of catchments, we divided the complete set into four subsets (of equal sizes) depending on streamflow autocorrelation, an index which provides information on the way catchments behave: catchments with flash floods have a low streamflow autocorrelation, while catchments with slow variations of streamflow present a much higher autocorrelation. For the same reason, forecasts are issued for different lead times: we assessed our results for 1-, 3-, 6-, 12-, 18-, 24-, 36- and 48-h lead times.

\subsection{Tested continuous approach}

We first tested the continuous approach: the model runs continuously for 1 year of warm-up (to obtain model states that are independent of the initial conditions) then continuously for the 4-year validation period. A previous analysis of our catchments (not shown here) demonstrated that a year of warming up is sufficient to reach states that no longer depend on the initial values for the studied catchments.

\subsection{Tested event-based approaches}

As the routing store of the selected model is updated using observed flow, the difference between the continuous and event-based approaches lies in the need to initialize the SMA store when working on an event basis. Three different simple event-based initializations are tested. For all of them, the performance criteria are computed over the same 4-year validation period so that they can be directly compared to the values obtained by the continuous approach.

\subsubsection{Poor-man's initialization}

The simplest initialization of the production store level is to choose an arbitrary value and then to run the model on a very short pre-forecast period ( 5 days) before issuing the forecast. 

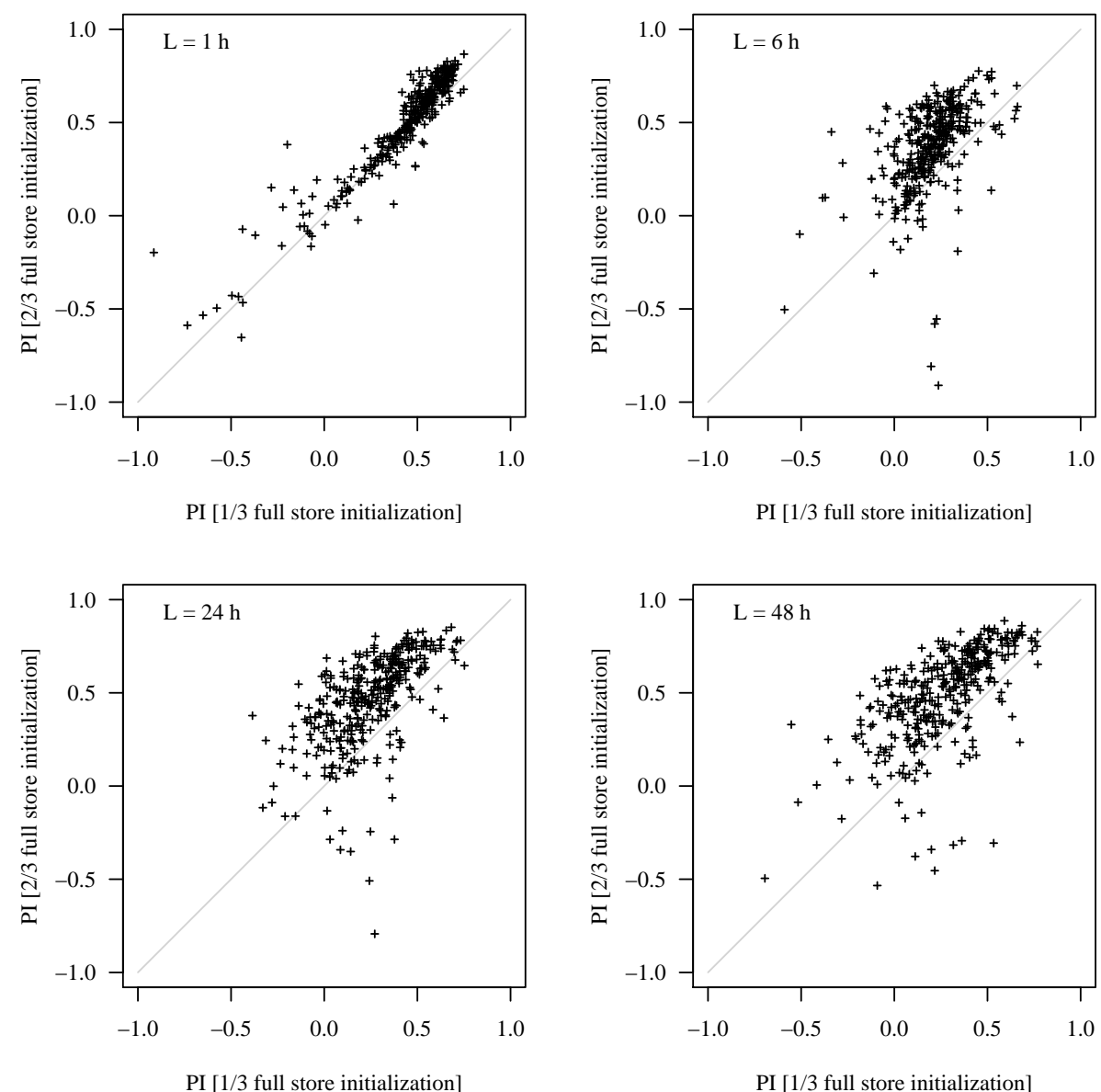

Fig. 3. Persistence indexes for two Poor-man's initializations at different initial values: the level of the SMA store is initially set at one-third or two-thirds of its capacity. Different initializations lead to very different performances.

Different values of the SMA store level (zero, one, two and three thirds of its capacity) are tested. This option makes it possible to check that the forecasts are indeed sensitive to the initialization of this store level.

\subsubsection{Climatic initialization}

The second tested approach consists in initializing the level of the production store at its pluri-annual average value (as calculated on the calibration period, i.e. over 5 years) at the beginning of a very short simulation period preceding the date of the forecast issue. Different simulation period lengths (from 5 to 15 days) are considered.

\subsubsection{Antecedent moisture conditions initialization}

The third initialization procedure is more elaborate. It looks in the calibration period archive for the time step that has the most similar antecedent precipitation index (API) value to the API value of the time step $t$ at which we issue the fore- cast. The API (see e.g. Kohler and Linsley, 1951) is computed as follows:

$$
\operatorname{API}(t)=\sum_{i=0}^{N_{\alpha}} \alpha^{i} P_{t-i}
$$

where $\alpha$ is a decay rate, $N_{\alpha}$ is the number of antecedent time steps taken into account and $P_{t-i}$ is the precipitation at time step $t-i . \quad N_{\alpha}$ is chosen to ensure that $\alpha^{i} P_{t-i}$ would be negligible compared to any precipitation $P_{t}$ for any $i$ higher than $N_{\alpha}$. Different values of $\alpha$ from $1-10^{-1}$ to $1-10^{-5}$ were tested. An $\alpha$ value of $1-10^{-3.5}$ was selected here as it gave the best results. Detailed results are not shown for the sake of simplicity.

\section{Results and discussion}

\subsection{Results on the whole catchment set}

First the Poor-man's initialization showed wide performance differences depending on the initial conditions for the tested 

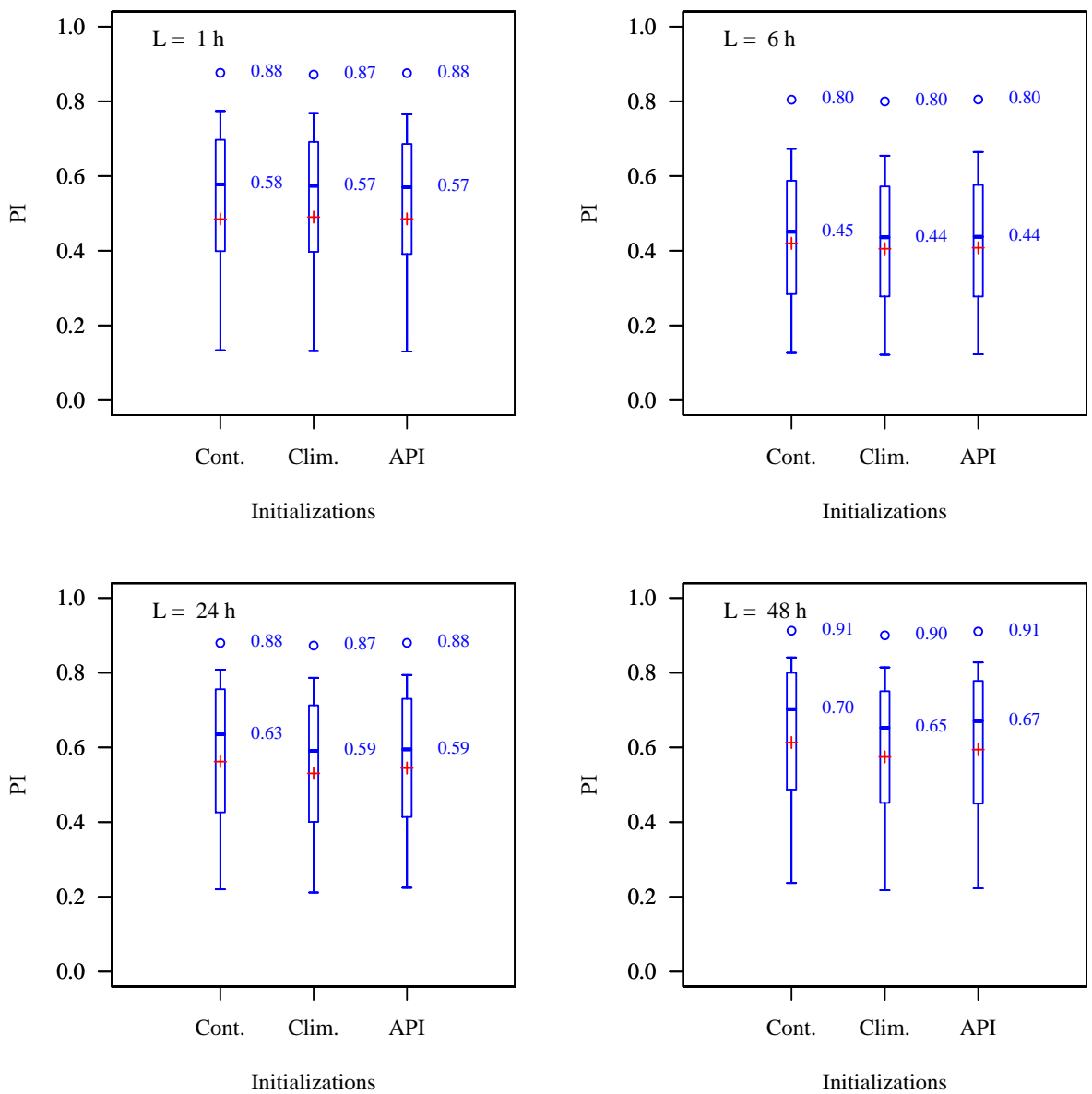

Fig. 4. Performance (persistence index) obtained by the model on 178 catchments, according to the initialization modes: the continuous strategy is depicted on the left and two event-based strategies (climatic and API strategies) are on the depicted right. The results are displayed for lead times ranging from 1 to $48 \mathrm{~h}$. The pre-forecast period for event-based strategies lasts $120 \mathrm{~h}$ ( 5 days). Boxplots give minimum and maximum values (dots) as well as 0.05 and 0.95 quantiles (whiskers), 0.25, 0.50, 0.75 quantiles (boxes). Mean values are indicated by crosses.

model and our catchments (Fig. 3). Thus, at the 1-h lead time, the persistence differences for different (arbitrary) initial values are greater than 0.03 (which is a significant difference) on more than $75 \%$ of the catchments; for the 48 -h lead time, this difference is greater than 0.14 for more than $90 \%$ of the catchments.

The results clearly show that the continuous approach gives the best results (see Table 1 and Figs. 4 and 5), and that the longer the lead time, the greater the difference in performance. Our interpretation is the following: model states do not reflect reality directly but are distorted representations of the real world as seen by the model. It is a better choice to initialize the model states as if they were seen from a given reality (continuous approaches) by the model rather than to impose values derived from measurements that do not correspond to the inner model logic. These results can be compared to those presented by Anctil et al. (2004a) and Merz and Blöschl (2009): antecedent soil moisture is a better control on the runoff coefficient ratio than antecedent precipitation depth and the state of a conceptual rainfall-runoff model can give valuable information on catchment moisture state.

The model has small time-to-peak errors: even for a $48-\mathrm{h}$ lead time, the time-to-peak errors of the model on more than $90 \%$ of the catchments are smaller than $5 \mathrm{~h}$. No significant difference in time-to-peak errors can be noted, whatever initialization method is considered (Table 2). Event-based initialization can even lead to very slightly smaller time-to-peak error than the continuous approach. Indeed, the initialization of the SMA store has a much more important influence on the simulated flood volume than on its timing. This can be seen on an example (Tarn River at Millau, spring 2004 floods, Fig. 6c): the different initializations lead to very different discharge magnitudes, but they all have the same temporal behaviour. 

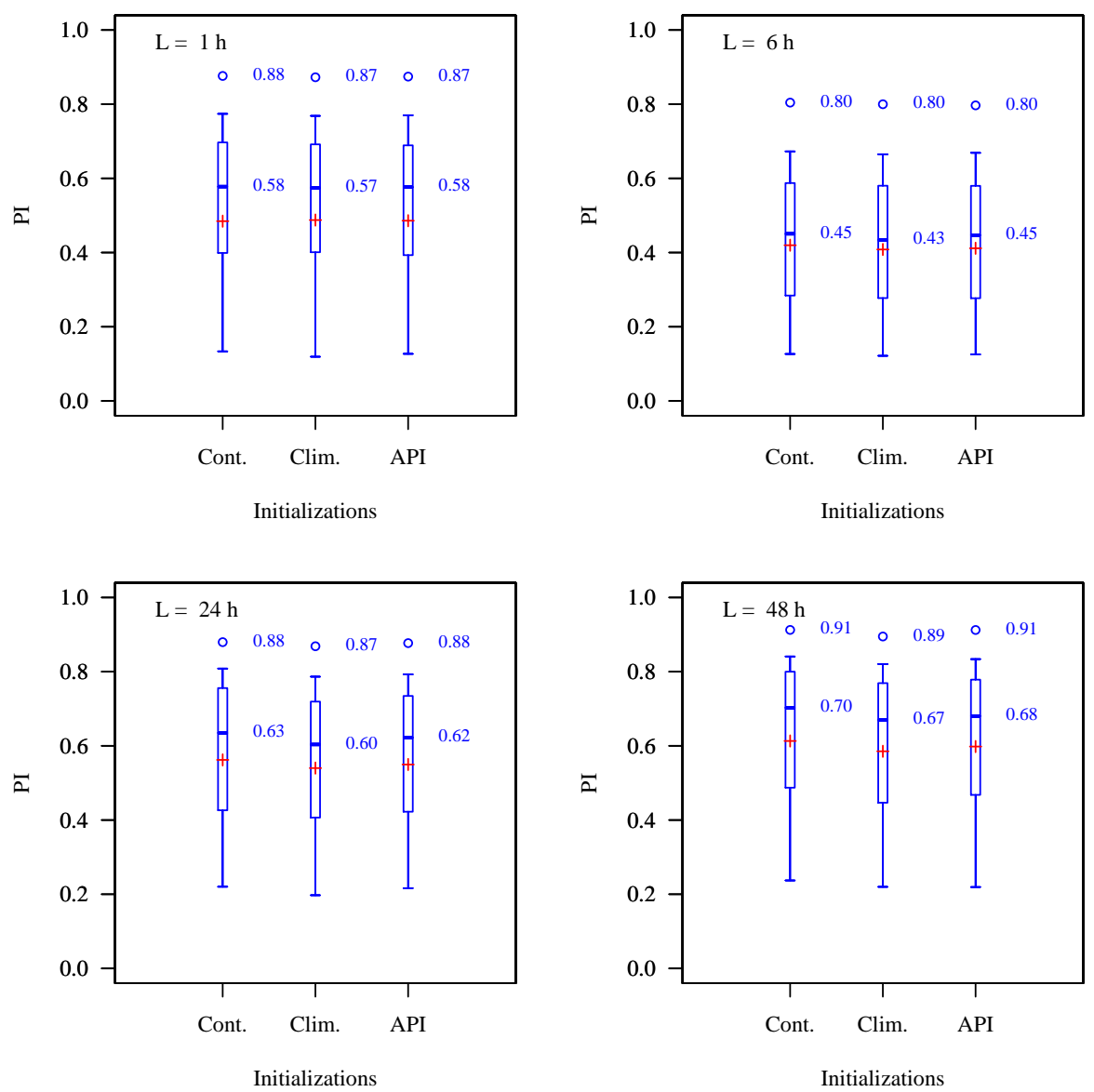

Fig. 5. Performance (persistence index) obtained by the model on 178 catchments, according to the initialization modes: continuous or event-based (climatic and API) strategies with a pre-forecast period lasting $360 \mathrm{~h}$. Results are displayed for lead times ranging from 1 to $48 \mathrm{~h}$.

Table 1. Medians of persistence index values obtained using the GRP model on the 178 catchment set with different initialization approaches for 1-, 6-, 24- and 48-h lead times.

\begin{tabular}{lcccc}
\hline Initialization & 1-h lead time & 6-h lead time & 24-h lead time & 48-h lead time \\
\hline Continuous & 0.58 & 0.45 & 0.63 & 0.70 \\
Best Poor man's & 0.56 & 0.40 & 0.50 & 0.64 \\
Best climatic & 0.57 & 0.44 & 0.61 & 0.67 \\
Best API-based & 0.58 & 0.45 & 0.62 & 0.68 \\
\hline
\end{tabular}

As expected, the event-based initialization strategies lead to poorer forecasting performances. However the good news (from an operational point of view) is that the performance loss due to the use of a simple event-based initialization strategy is not large for most catchments (see Figs. 4 and 5). This result must be moderated by the fact that we used controlled offline data which are supposed to be high quality compared to the data used in real-time operations. Event-based initialization can not always be done with sufficiently controlled data and therefore might encounter more errors in rainfall while the continuous approach may do a better use of controlled data.

The event-based initialization strategies we tested ranked in a quite logical manner: the best one is not surprisingly the API method, which is the most informative approach concerning the catchment initial moisture conditions. Then comes the climatic solution, which provides little information. The strategy that leads to the lowest performances is the Poor-man's approach. 
Tarn river at Millau Spring 2004 events Lead time: $6 \mathrm{~h}$

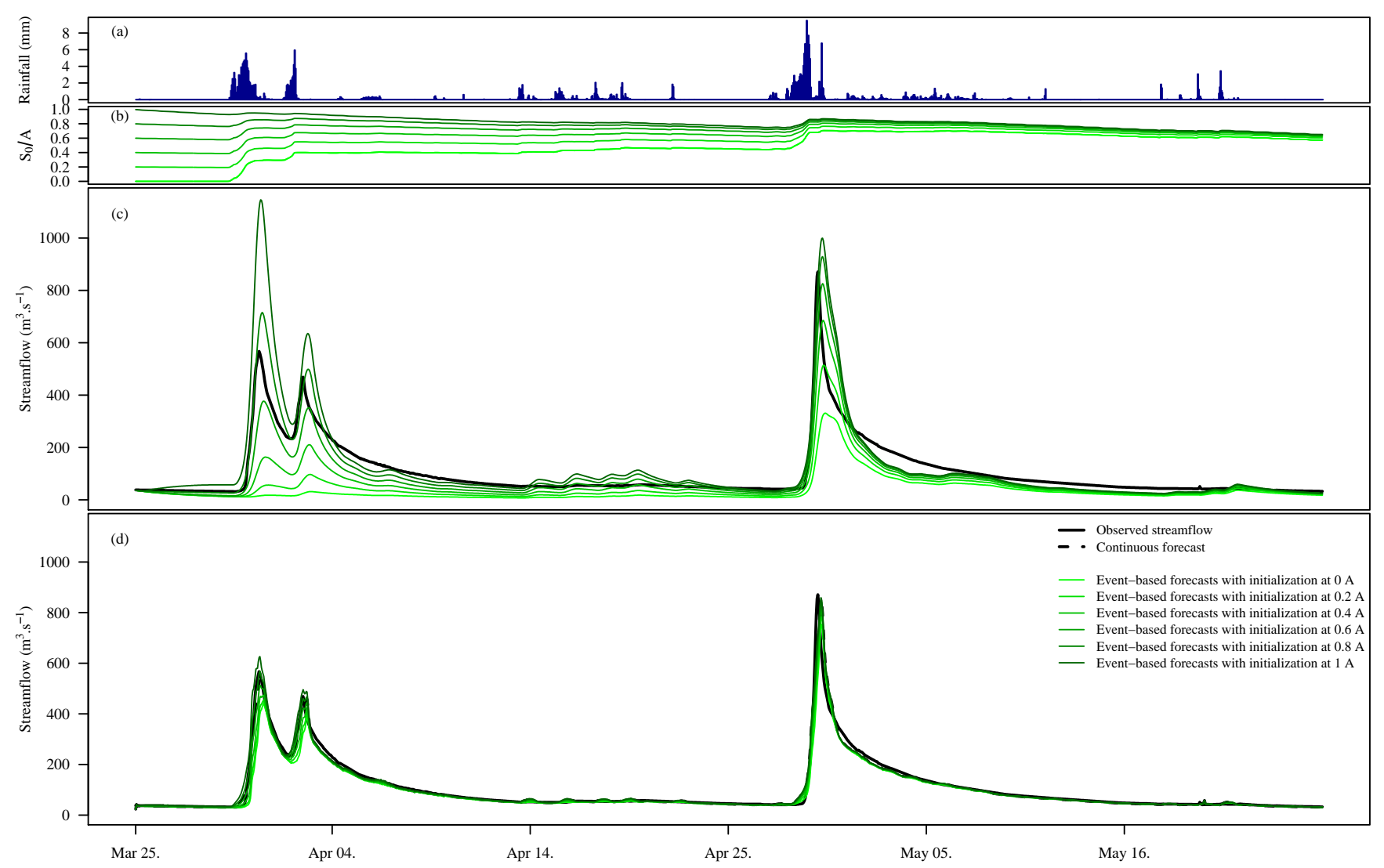

Fig. 6. (a) Precipitation during spring 2004 events. (b) Changes in the production store level after its initialization at different values on the 25 March 2004: there is convergence mainly during showers. Different initializations lead to very different forecasts when no updating technique is applied (c), whereas forecasts depend much less on the initial production store content when the model is updated (d).

Table 2. Mean time-to-peak errors obtained with the different initialization methods (expressed in hours)

\begin{tabular}{lcccc}
\hline Initialization & 1-h lead time & 6-h lead time & 24-h lead time & 48-h lead time \\
\hline Continuous & 0.23 & 1.39 & 0.69 & -0.09 \\
Best Poor man's & 0.25 & 1.42 & 0.76 & -0.05 \\
Best climatic & 0.24 & 1.43 & 0.73 & -0.09 \\
Best API-based & 0.24 & 1.45 & 0.71 & -0.01 \\
\hline
\end{tabular}

Those results are indeed model-dependent: they depend actually on the model structure used and on the choice of the updating methods. However, Moore (2007) pointed out that many models present strong structural and behavioural similarities; consequently the conclusions may remain valid for many conceptual models. Conversely, the choice of the updating method is crucial: updating the SMA store leads to very poor performance with the same model structure (Javelle and Berthet, 2008; Tangara, 2005).

\subsection{Use of a pre-forecast period: a compromise ap- proach?}

Actually, many so called event-based approaches are not purely event-based since they use a short pre-forecast period on which the model is run before issuing the forecast: the model is initialized at time step $t-\tau$ and run during the pre-forecast period from $t-\tau$ to $t$ before being used to issue a forecast at $t$ of the runoff values at $t+1, \ldots, t+L$. Many event-based approaches consider initial conditions to 
Table 3. Medians of persistence index values obtained by the selected model with the climatic initialization approach using pre-forecast period of different lengths for 1-, 6-, 24- and 48-h lead times. HU length ranges from 1 to $64 \mathrm{~h}$ depending on the catchment.

\begin{tabular}{lcccc}
\hline Pre-forecast length & 1-h lead time & 6-h lead time & 24-h lead time & 48-h lead time \\
\hline HU length & 0.57 & 0.42 & 0.57 & 0.64 \\
5 days & 0.57 & 0.44 & 0.59 & 0.65 \\
10 days & 0.57 & 0.44 & 0.61 & 0.66 \\
15 days & 0.57 & 0.43 & 0.60 & 0.67 \\
Continuous & 0.58 & 0.45 & 0.63 & 0.70 \\
\hline
\end{tabular}
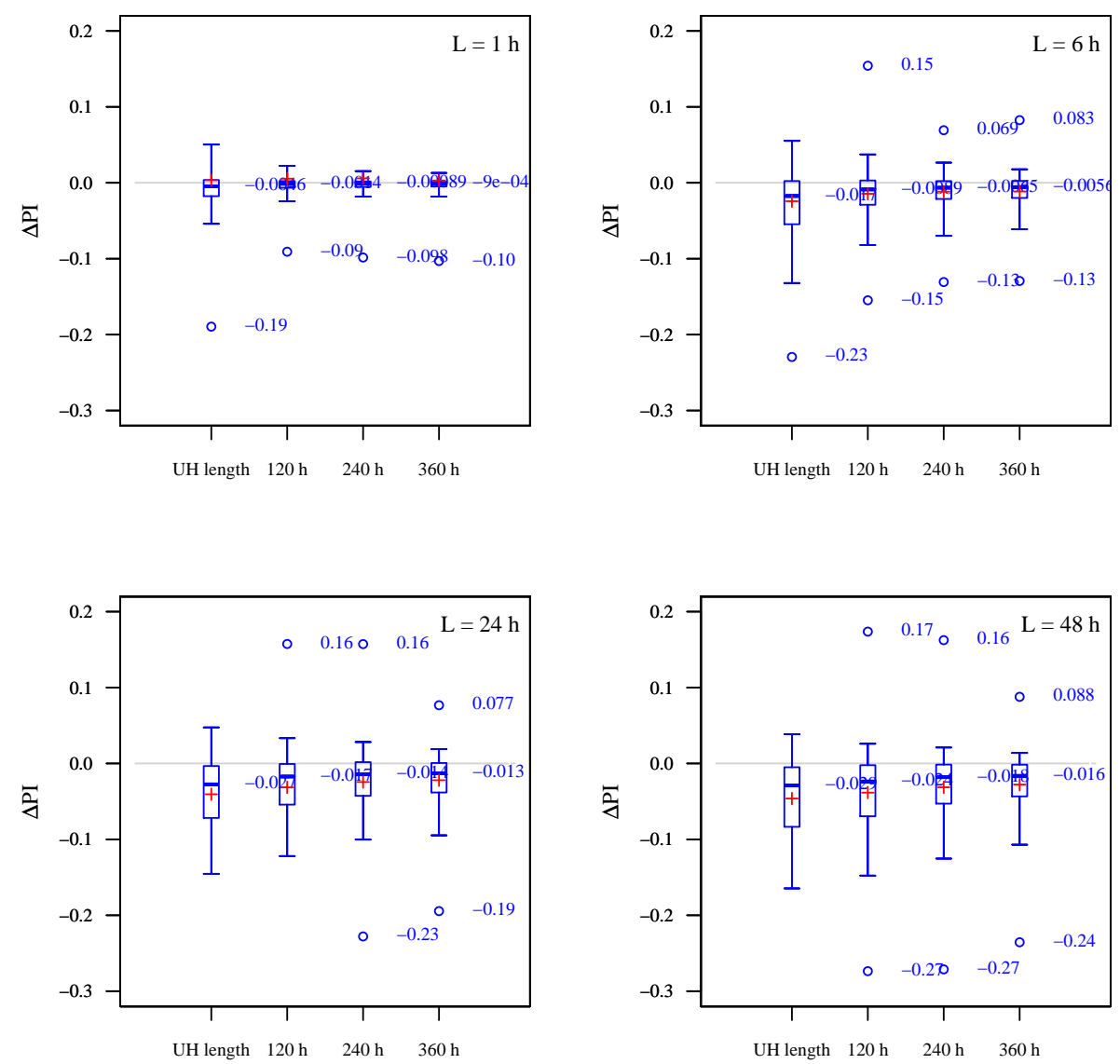

Fig. 7. Differences between the performance (persistence index) obtained by the model on 178 catchments in continuous mode and using an event-based climatic initialization, according to the pre-forecast period length: the longer the pre-forecast period, the smaller the differences.

be parameters; this requires pre-forecast period data to calibrate the initial conditions. These models can not really be considered as purely event-based. Thus, some modellers, e.g. Merz and Bárdossy (1998) and Sheikh et al. (2009), chose to use an inter-event model to initialize the most sensitive states. Another example is given by Anctil et al. (2004a), who used artificial neural networks (ANN) for flow forecasting on two catchments: they showed that a long-term soil moisture index derived from a continuous model is a valu- able input which improves forecasts. Here again the resulting models are not purely event-based: they belong to a continuum between event-based and continuous models.

In this paper, our initialization strategies also use a short pre-forecast period to come closer to a continuous approach: the resulting initial conditions are a mix of assumptions implied by the initial choice and of the model's internal representation of the catchment behaviour. 

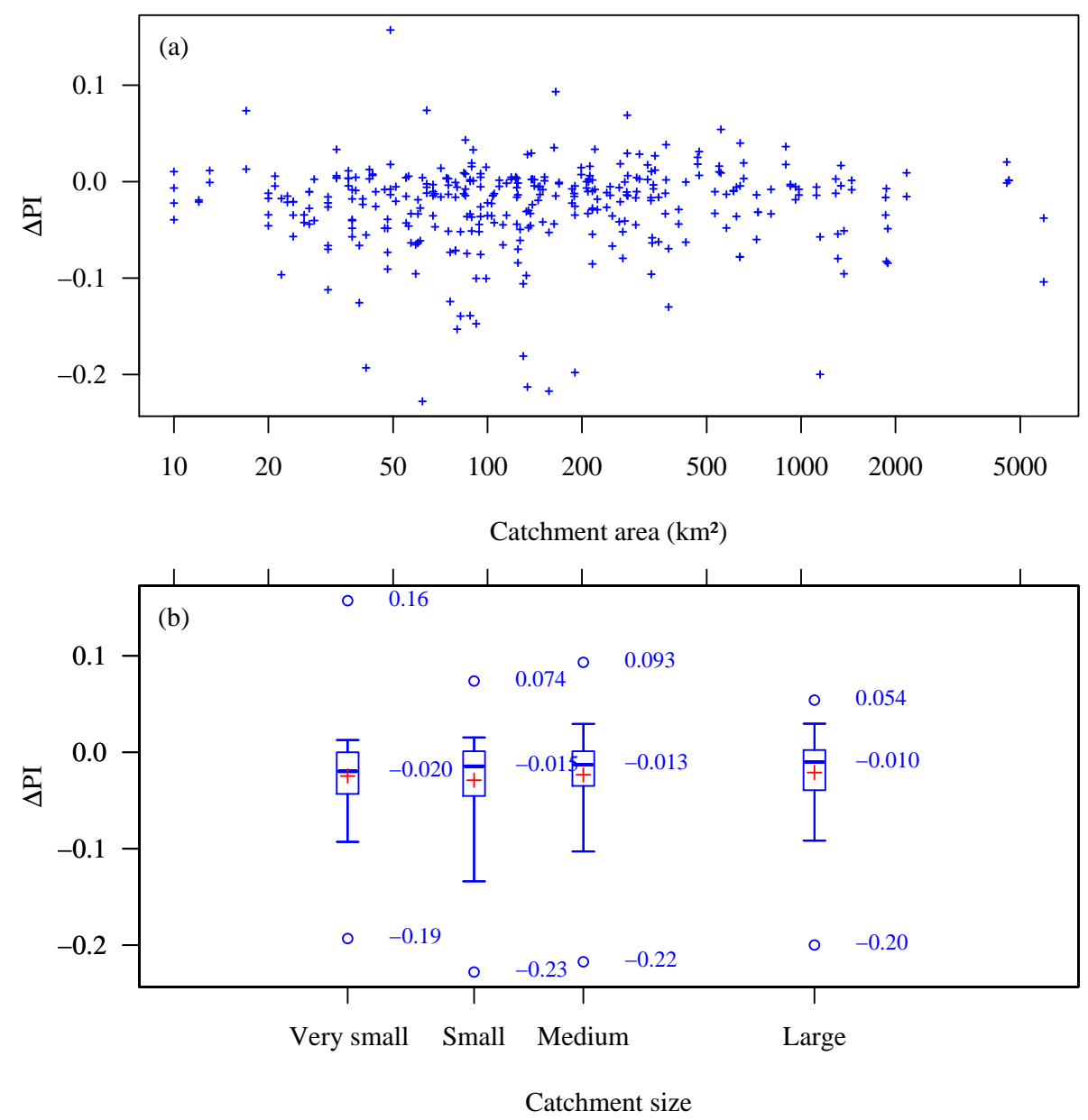

Fig. 8. Differences between the performance (persistence index) obtained by the model on 178 catchments in continuous mode and using an event-based climatic initialization approach, depending on the catchments' areas (a). Here lead time is $6 \mathrm{~h}$. The pre-forecast period for the event-based strategy lasts $240 \mathrm{~h}$. Four classes of catchments are defined: the first class groups the $25 \%$ smallest catchments, the second class, the following $25 \%$, etc. Distributions of the difference in performance for every class are displayed (b).

In the strategies we tested, this pre-forecast period lasts at least the length of the UH (to obtain proper values in the $\mathrm{UH})$ and was tested up to 15 days. It is clear for all eventbased initializations that the longer the pre-forecast period, the better the performance (Fig. 7). For the climatic initialization, a pre-forecast period of 5 days leads to performance significantly lower than the performance obtained with a continuous initialization. However, a 15-day pre-forecast period allows performances close to what is given by the continuous approach (see Table 3).

\subsection{Do results depend on catchment size?}

Figure 8 shows the difference in performances obtained by the same model running in continuous mode and in eventbased mode (with climatic initialization) depending on the catchment area. No clear trend can be detected from these analyses. It is interesting to note moreover that the model's performance does not decrease as the catchment size increases: the catchment behaves as a low-pass filter (Oudin et al., 2005) and as the catchment size increases, forecasting in fact becomes an easier task (for a given lead time).

\subsection{Do results depend on catchment reactivity?}

We found no relationship between catchment response time and the impact of choosing a continuous or an event-based strategy. Figure 9 shows the difference in performances obtained by the same model running in continuous mode and in event-based mode (with climatic initialization) depending on the catchments discharge autocorrelation. No trend was detected from these analyses. 

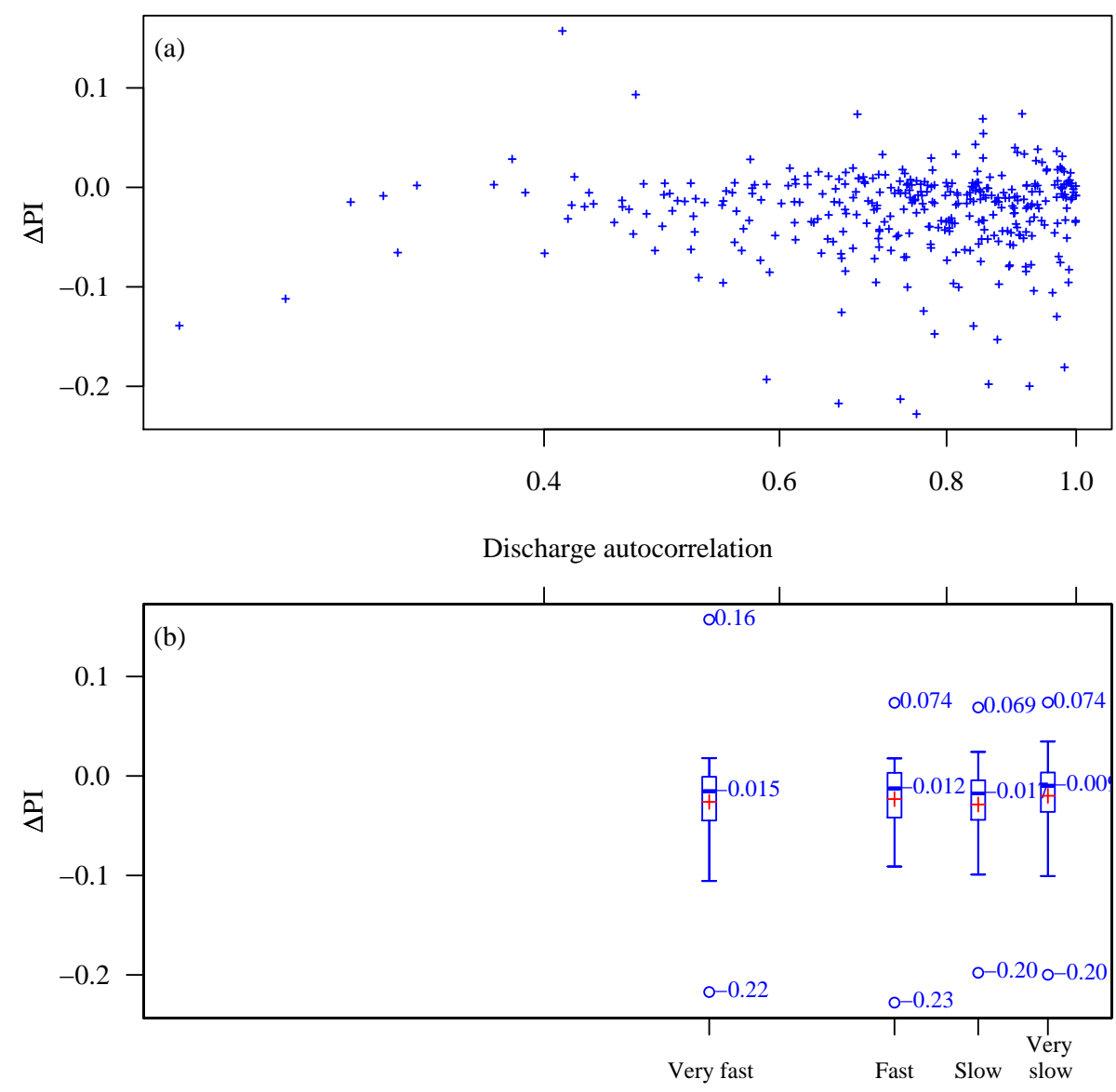

Catchment reactivity

Fig. 9. Differences between the performance (persistence index) obtained by the model on 178 catchments in continuous mode and using an event-based climatic initialization approach, depending on the discharge autocorrelation (a). Here the lead time is $6 \mathrm{~h}$. The pre-forecast period for the event-based strategy lasts $240 \mathrm{~h}$. Four classes of catchments are defined: the first class groups the $25 \%$ of catchments with the lowest discharge autocorrelation, the second class, the following $25 \%$, etc. Distributions of the difference in performance for every class are displayed (b).

\subsection{Impacts of the updating procedure}

When used to issue discharge forecasts, hydrological models are most often updated (Refsgaard, 1997). In practice, this means that the discharge forecast no longer depends on forcing variables only (e.g. precipitation, evapotranspiration, etc.) but also on the information contributed by the data assimilation process. The discharge forecast is constrained by data assimilation and consequently it may depend (much) less on the internal states and so on their initialization.

We used the GRP model with and without updating techniques to compare the influence of initialization of the model's SMA store (which is never updated) on forecasts in both cases. Figure 6 shows an example of spring floods for the Tarn River at Millau $\left(2170 \mathrm{~km}^{2}\right)$. We chose different initial production store levels from 0 (empty store) to its maximum capacity $A$ (full store). From those initial values, the production store levels converge slowly; convergence is mostly achieved during showers (Fig. 6a, b). The differences in production store levels lead to dramatic differences in discharge forecasts when no updating technique is applied (Fig. 6c), whereas the 6-h forecasts are much more constrained with data assimilation (Fig. 6d). Similar results were obtained for the other catchments of our data set.

Thus, the updating procedure used in forecasting models does limit the impact of crude initialization procedures, in comparison with simulation models: this explains why a rather simple procedure with a short pre-forecast period gives results close to those obtained when using a continuous initialization. 


\section{Synthesis and conclusions}

Initial conditions are known to be of crucial importance for hydrological models. In this paper, we compared different initialization strategies of the soil moisture component of a rainfall-runoff forecasting model. The continuous mode was compared to several event-based approaches for the same model. The main conclusion is that the best results were obtained when the model was run in a continuous mode. This corroborates the results of previous studies (e.g., Anctil et al., 2004b). However, we showed that one of our tested eventbased initialization strategies (i.e., the API-based initialization) could lead to performances rather close to what is obtained with the continuous approach, provided that the model can be run on a short pre-forecast period.

Indeed, the sensitivity of the model outputs to the initial conditions is much lower in forecasting mode than in simulation mode (i.e. without updating through the assimilation of measured streamflow): the output is considerably constrained by the information contributed during the observed flow assimilation process, which partly compensates for the errors in initial values.

Given the large and varied data set used here, we believe that these results are not catchment-dependent (in particular we found no relation to catchment size or reactivity). The results may remain to some extent model-dependent. However, we expect that the behaviours we observed can also be found for many forecasting models, since they all have to use efficient data assimilation.

The loss in performance when running the model using event-based strategies is not substantial: indeed, in most cases, the difference is not really significant. This means that if an efficient assimilation of the last observed streamflow is possible, event-based strategies can be efficiently used for operational purposes when and where it is impossible to run a model continuously.

Acknowledgements. This work is partly funded by a research grant from the French Ministry of Agriculture. The authors gratefully acknowledge the assistance of Nicolas Le Moine (Cemagref) for data collection and Météo France for providing meteorological data as well as French and Quebec operational Flood Forecasting Services for fruitful discussions. The authors also thank Uwe Ehret and Julien Lerat and two anonymous reviewers for their comments which led to improvements in the article.

Edited by: N. Verhoest

\section{References}

Amengual, A., Diomede, T., Marsigli, C., Martín, A., Morgillo, A., Romero, R., Papetti, P., and Alonso, S.: A hydrometeorological model intercomparison as a tool to quantify the forecast uncertainty in a medium size basin, Nat. Hazards Earth Syst. Sci., 8, 819-838, 2008, http://www.nat-hazards-earth-syst-sci.net/8/819/2008/.

Anctil, F., Michel, C., Perrin, C., and Andréassian, V.: A soil moisture index as an auxiliary ANN input for stream flow forecasting, J. Hydrol., 286, 155-167, 2004a.

Anctil, F., Perrin, C., and Andréassian, V.: Impact of the length of observed records on the performance of ANN and of conceptual parsimonious rainfall-runoff forecasting models, Environmental Modelling \& Software, 19, 357-368, 2004b.

Andréassian, V., Hall, A., Chahinian, N., and Schaake, J.: Introduction and Synthesis: Why should hydrologists work on a large number of basin data sets?, IAHS-AISH Publication, 307, 1-5, 2006.

Aubert, D., Loumagne, C., and Oudin, L.: Sequential assimilation of soil moisture and streamflow data in a conceptual rainfall Runoff model, J. Hydrol., 280, 145-161, 2003.

Box, G. E. P. and Jenkins, G. M.: Time Series Analysis: Forecasting and Control, Holden Day Inc., Oakland, California, USA, 575 pp., 1976.

Brocca, L., Melone, F., Moramarco, T., and Singh, V. P.: Assimilation of Observed Soil Moisture Data in Storm Rainfall-Runoff Modeling, J. Hydrol. Eng., 14, 153-165, doi:10.1061/(ASCE) 1084-0699(2009)14:2(153), 2009.

Cemagref: Inventory and diagnosis of simple existing flood forecasting models on the Seine River basin (in French), Final report 133 pages, Hydrosystems and Bioprocesses Research Unit, DIREN Île-de-France, Antony, France, 2005.

Da Ros, D. and Borga, M.: Adaptive use of a conceptual model for real time flood forecasting, Nord. Hydrol., 28, 169-188, 1997.

Dietrich, J., Trepte, S., Wang, Y., Schumann, A. H., Voß, F., Hesser, F. B., and Denhard, M.: Combination of different types of ensembles for the adaptive simulation of probabilistic flood forecasts: hindcasts for the Mulde 2002 extreme event, Nonlinear Proc. Geophys., 15, 275-286, 2008.

Javelle, P. and Berthet, L.: Inter-comparaison des modèles de prévision des crues développés au Cemagref: GR3H et GR3P, convention 2008 Cemagref / Ministère de l'Écologie et du Développement durable - service central d'hydrométéorologie et d'appui la prévision des inondations, Rapport Cemagref, 23 pp. (in French), 2008.

Kitanidis, P. and Bras, R.: Real-time forecasting wih a conceptual hydrologic model. 1. Analysis of uncertainty, Water Resour. Res., 16, 1025-1033, 1980a.

Kitanidis, P. and Bras, R.: Real-time forecasting with a conceptual hydrologic model. 2. Applications and results., Water Resour. Res., 16, 1034-1044, 1980b.

Klemeš, V.: Operational testing of hydrologic simulation models, Hydrol. Sci. J., 31, 13-24, 1986.

Kohler, M. A. and Linsley, R. K. J.: Predicting runoff from storm rainfall, Res. Paper, 34, US Weather Bureau, Washington DC, USA, 1951.

Lamb, R. and Kay, A.: Confidence intervals for a spatially generalized, continuous simulation flood frequency model for Great Britain, Water Resour. Res., 40, W07501, 
doi:10.1029/2003WR002428, 2004.

Le Moine, N.: Le bassin versant de surface vu par le souterrain: une voie d'amélioration des performances et du réalisme des modèles Pluie - Débit?, Ph.D. thesis, Université Pierre et Marie Curie (Paris VI), 2008.

Linsley, R.: Proceedings of the international symposium on rainfallrunoff modelling, in: Proceedings of the international symposium on rainfall-runoff modelling, edited by: Singh, V., 3-22, Water Resources Publications, Littleton, CO, USA, 1982.

Merz, B. and Bárdossy, A.: Effects of spatial variability on the rainfall runoff process in a small loess catchment, J. Hydrol., 212213, 304-317, 1998.

Merz, R. and Blöschl, G.: A regional analysis of event runoff coefficients with respect to climate and catchment characteristics in Austria, Water Resour. Res., 45, W01405, doi:10.1029/2008WR007163, 2009.

Moore, R., Cole, S., Bell, V., and Jones, D.: Issues in flood forecasting: Ungauged basins, extreme floods and uncertainty, in: IAHS-AISH Publication no. 305, 103-122, 2006.

Moore, R. J.: The PDM rainfall-runoff model, Hydrol. Earth Syst. Sci., 11, 483-499, 2007, http://www.hydrol-earth-syst-sci.net/11/483/2007/.

Moore, R. J., Bell, V. A., and Jones, D. A.: Forecasting for flood warning, Comptes Rendus Geosciences, 337, 203-217, http://www.sciencedirect.com/science/article/ B6X1D-4F0199D-6/2/c3a587ebec49a67b3ff4217a8dfab536, 2005.

Morton, F.: Operational estimates of areal evapotranspiration and their significance to the science and practice of hydrology, J. Hydrol., 66, 1-76, 1983.

Nalbantis, I.: Use of multiple-time-step information in rainfallrunoff modeling, J. Hydrol., 165, 135-159, 1995.

Nalbantis, I.: Real-time flood forecasting with the use of inadequate data, Hydrol. Sci. J., 45, 269-284, 2000.

National Research Council (NRC): Report of a Workshop on Predictability \& Limits-To-Prediction in Hydrologic Systems, 0309-08347-8, National Academic Press, Washington DC, USA, 118 pp., 2002.

Norbiato, D., Borga, M., Degli Esposti, S., Gaume, E., and Anquetin, S.: Flash flood warning based on rainfall thresholds and soil moisture conditions: An assessment for gauged and ungauged basins, J. Hydrol., 362, 274-290, 2008.

Noto, L., Ivanov, V., Bras, R., and Vivoni, E.: Effects of initialization on response of a fully-distributed hydrologic model, J. Hydrol., 352, 107-125, 2008.
Ogrosky, H. O. and Mockus, V.: in Handbook of Applied Hydrology, chap. Hydrology of Agricultural Lands, 21:1-21:97, McGraw-Hill inc., USA, 1964.

Oudin, L., Hervieu, F., Michel, C., Perrin, C., Andréassian, V., Anctil, F., and Loumagne, C.: Which potential evapotranspiration input for a lumped rainfall-runoff model? Part 2 - Towards a simple and efficient potential evapotranspiration model for rainfallrunoff modelling, J. Hydrol., 303, 290-306, 2005.

Perrin, C., Michel, C., and Andréassian, V.: Improvement of a parsimonious model for streamflow simulation, J. Hydrol., 279, 275289, 2003.

Refsgaard, J. C.: Validation and Intercomparison of Different Updating Procedures for Real-Time Forecasting, Nord. Hydrol., 28, 65-84, 1997.

Refsgaard, J. C. and Henriksen, H. J.: Modelling guidelines - terminology and guiding principles, Adv. Water Resour., 27, 71-82, 2004.

Refsgaard, J. C., Thorsen, M., Jensen, J., Kleeschulte, S., and Hansen, S.: Large scale modelling of groundwater contamination from nitrate leaching, J. Hydrol., 221, 117-140, 1999.

Shamseldin, A. Y.: River Basin Modelling for Flood Risk Mitigation, chap. Real-time river flow forecasting, Taylor \& Francis/Balkema, Leiden, the Netherlands, 181-195, 2006.

Sheikh, V., Visser, S., and Stroosnijder, L.: A simple model to predict soil moisture: Bridging Event and Continuous Hydrological (BEACH) modelling, Environ. Model. Softw., 24, 542-556, 2009.

Tan, S., Chua, L., Shuy, E., Lo, E.-M., and Lim, L.: Performances of rainfall-runoff models calibrated over single and continuous storm flow events, J. Hydrol. Eng., 13, 597-607, 2008.

Tangara, M.: Nouvelle méthode de prévision de crue utilisant un modèle pluie-débit global, Ph.D. thesis, École pratique des hautes études de Paris, 2005.

Vieux, B., Cui, Z., and Gaur, A.: Evaluation of a physics-based distributed hydrologic model for flood forecasting, J. Hydrol., 298, 155-177, 2004.

Vivoni, E., Entekhabi, D., Bras, R., and Ivanov, V.: Controls on runoff generation and scale-dependence in a distributed hydrologic model, Hydrol. Earth Syst. Sci., 11, 1683-1701, 2007, http://www.hydrol-earth-syst-sci.net/11/1683/2007/.

Zehe, E. and Blöschl, G.: Predictability of hydrologic response at the plot and catchment scales: Role of initial conditions, Water Resour. Res., 40, W10202, doi:10.1029/2003WR002869, 2004. 